\title{
loT-based Smart Lab System in Schools using Arduino and Bluetooth based Android Smartphone
}

\author{
Safaa M. Elatawy \\ Dept. of Computer Science, \\ Faculty of Specific Education \\ Damietta University, Egypt
}

\begin{abstract}
The Internet of Things (IoT) allows interaction between equipment and applications, as it provides a platform that allows devices to be connected, data collected and controlled remotely. This work aims to suggest an IoT-based system to control general activities in the laboratory room. Including monitor the lighting, temperature, humidity, fire, and gas intensity, control door \& windows at lab and has an infrared sensor to ensure lab security. The proposed model uses some tools such as the Adriano UNO chips, a Bluetooth connection, a temperature sensors, humidity sensors, Motion sensors, Lighting sensor, gas sensors and Servo motor, some bulbs, wires and connectors, various resistors, and Crystal led screen. The mobile application used as controller. All components are installed on the mini-model of the lab. This component was connected to the Bluetooth part and the mobile phone. This approach combines between software and hardware technologies. The results indicate that a mobile phone can be configured as a central unit to control the functionality of activity patterns that can be planned and determined in advance. It can also control different parts of the lab and automatically react to hazards such as fires and gas leaks.
\end{abstract}

\section{General Terms}

Internet of Things (IoT), Arduino, Mobile Application

\section{Keywords}

IOT, android, smart lab, Arduino, Bluetooth, Mobile Communication. Sensors

\section{INTRODUCTION}

Nowadays, Internet of Things (IOT) is the booming research topic, can be defined to link a group of surrounding elements and smart devices, which exchange information between them without human intervention [1]. With the huge development of technology in communications, cloud computing, and the Internet, iot has emerged to include many of the things we see in our environment. all of this things are connected with the internet by several methods, like Bluetooth, Wi-Fi and ZigBee 4G, 5G, (and etc....) [2]. The Internet of Things needs a large group of tools such as sensors, Wireless Sensor Networks, RFID, etc....[3]. A long time ago, the sensors were used to monitor the working environment and the devices. However, connecting these sensors to the Internet and using them to collect data caused a revolution in various fields, from industry management, healthcare retail, transportation, education and others [4]. Because of the developments in the field of artificial intelligence and the integration of sensors in mobile phones, applications for interactions based on mobile devices with devices have increased, leading to the spread of Internet of things devices in all fields [5]. Including smart city systems [6-8], smart home systems [9,10], that allow remote control of home appliances and lighting using mobile applications, surface cleaning Robots[11], and etc... . The solutions based on the Internet of things have gained a demand for marketing and increased competition for their design and innovation.

School laboratories are the places where practical experiments are conducted, which are considered one of the most important educational tools within the school environment, especially as they are used to teach difficult concepts $[12,13]$. At present, the laboratories include many smart devices such as smart boards, data show devices, tabletop devices to, healthy chairs, computer devices, Air conditioners, devices and tools using in Experimentation. All these different smart tools are for better educational benefits of school experiment by reducing the time spent on practical details and increasing the time available for interaction among students, and between students and teacher [14]. Environment of the lab is the one of the main aspect of a School laboratories, the temperature, humidity level, gas contamination on the air, security lab, lighting control, are necessary components in a lab for healthy and safe environment. On that perspective, this study presented IoTbased Smart lab System using Arduino and Bluetooth based android smartphone. This system can contain the Internet; the system can monitor the lighting, temperature, humidity, burglar buzzer fire, and gas intensity, control door \& windows at lab and has an infrared sensor to ensure lab security. The communication over internet from the device is the most essential component of an IoT system .The structure of this paper is following: section one is the introduction, section two related work, section three explains the system description, section four presents the method, results, finally section five includes conclusion and future work.

\section{RELATED WORK}

Smart automation systems is an important issue that many researchers have worked on. In [15] M. Poongothai and P. Muthu Subramanian, 2018 presented IoT system and mobile application to monitor the overall activities of the lab such as environmental parameters, utilization of devices, and energy consumption using sensors. To monitor the light intensity, temperature, and humidity in the laboratory, sensors, relays, current transformers, NESP8266, Arduino UNO, and Raspberry Pi3 were used. From the results of implementation were reducing their energy consumption considerably. In [16] Andrey V. Gavrilov, et al 2014 presented a smart school laboratory's architecture. Using Multi-Agent Systems and technologies of Smart Environment to refinement effectiveness during work in laboratory with any technological tools such as machine tools, computers, and so. In [17] Hu Yin ,2015 proposed A smart lab using mobile application, traditional IT laboratory, and IOT to present a practice platform to learn concepts of mobile application technology and IOT for university students. It aid to presented smart environment for a university to help improve campus management. In [18] Mary 
Cherian et al, 2014 presented a smart lab using wireless technologies. It used Passive infrared sensor and environment sensor to implementation of smart lab. Which include the Security module and ambient lighting module. However, the drawback is the limited resources. [19] Ala Al-Fuqaha et al, 2015 presented summary of the mostly relevant protocols using in IOT such as XMPP, MQTT, DDS, and AMQP to provide developers, and researchers, basis about how the various protocols fit jointly. [20] Yassein, M. B. et al, 2012 suggested a view of the various protocols RESTFUL; XMPP; CoAP; DSS; MQTT; AMQP. Which presented IoT services. Which using in the application layer to message passing.

From a literature survey it was observed that monitoring the school laboratory environment needs to improve the interaction between software and hardware in order to control the environmental condition of the laboratory and discover whether the laboratory is compatible to be a smart laboratory or not.

In this paper, we focused on the general environment for smart labs and for this, we used many sensors to monitor the environmental condition of school laboratories and achieve safety against theft .The overall atmospheric condition of the laboratory, from the level of temperature, humidity level, gas pollution, fire insurance, and laboratory safety against unwanted entry, all of these issues may be a source of concern for students, teachers, and management. This study proposes to uncover the environmental state of smart laboratories that contain sensors to obtain values in real time, secure the laboratory, and determine whether the laboratory condition is sufficient to conduct experiments and interaction between the students and teacher.

\section{SYSTEM ARCHITECTURE AND DESIGN}

\subsection{Sensors and Tools}

- Arduino UNO board

- Bluetooth unit

- $\quad$ Led Lighting control

- Gas sensor

- Motion sensor

- Humidity sensor

- Temperature sensor

- Servo motor

- Crystal led screen

- $\quad$ Some leds

- Female-to-Male - Female-to-Female Jumper Cables.

- Buzzer

- Usb Ac/ Dc power

- Android phone application

\subsection{System Design}

This system provides a low cost and flexible system for laborite control and environmental monitoring. In this system, the web server is used with IP devices to access and control devices remotely. The proposed system does not require a dedicated server computer. This system was designed by using suitable size components to modification in this system. The figure 1 shows All System architectures.

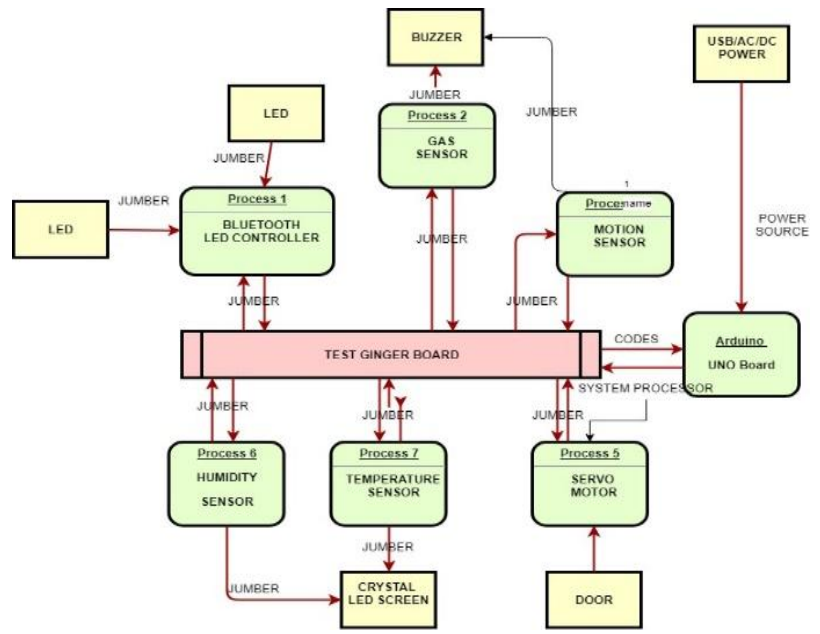

Fig. 1 the System architectures of the suggested smart lab

The figure 2 shows Block Diagram of the smart lab.

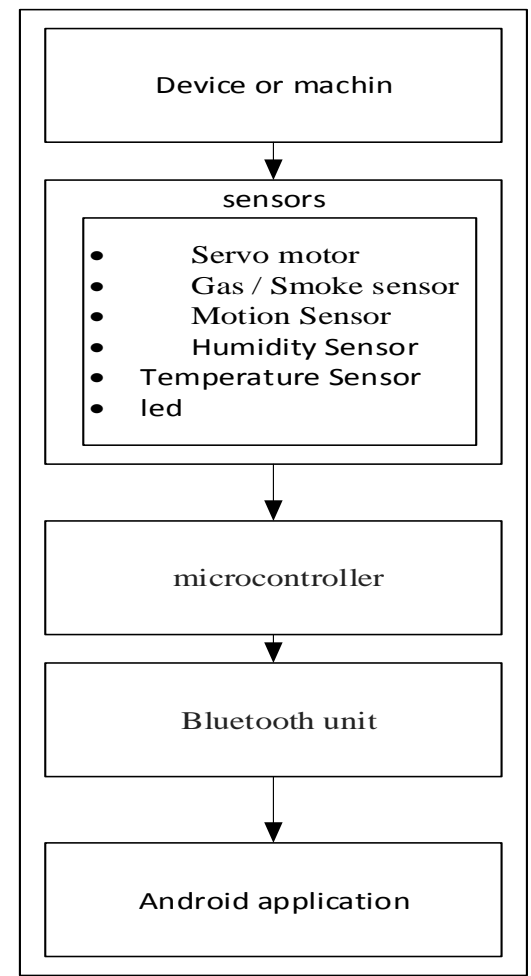

Fig. 2. The Block Diagram of the suggested system to smart lab

The system architecture consists the many parts As follows:

\subsubsection{The Arduino UNO R3}

The Arduino UNO R3 based on the ATmega328. It is a microcontroller board [21] as shown in figures.3. 


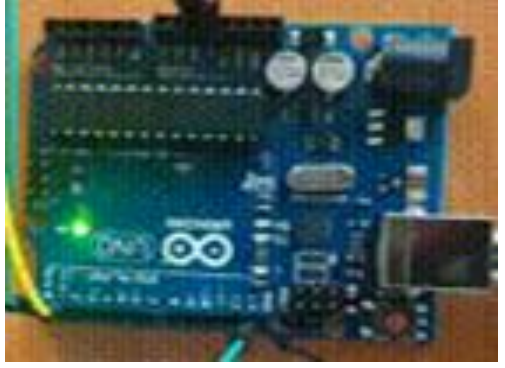

Fig3. Arduino Uno Board

The Arduino Uno is open source prototyping platform gives us flexibility in the types of different devices and software. it includes 14 digital pins, can be used 6 of them as analog inputs, PWM outputs can be used 6 as well, a power jack, a USB connection, a $16 \mathrm{MHz}$ ceramic resonator, and a reset button. It consist of everything approximately to support the microcontroller. To use Arduino UNO we need to power it with an AC-to-DC adapter, or connect it with a computer by a USB cable. The Advantage of Arduino UNO R3 is that it can be programmed as a USB to serial converter

\subsubsection{Bluetooth unite}

Bluetooth wireless technology is short-range radio technology for exchanging data. Uses UHF radio waves from mobile and fixed devices in the ISM range from 2.4 to $2.485 \mathrm{GHz}$ [22]. The Bluetooth unite being used allows us to transfer signals. It receives the signals from the Android application and transmits it to the serial port of the Arduino Uno R3. We used Bluetooth HC-05 module, which designed for wireless serial connection setup. The Bluetooth HC-05 is a master and slave. It uses CMOS technology with Adaptive Frequency Hopping Feature of CSR Blue core 04. Figure 4 shows the Arduino UNO R3 connection to Bluetooth HC-05.

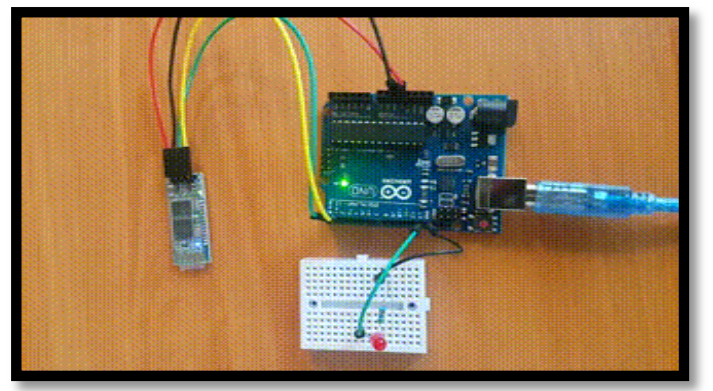

Fig 4. Arduino UNO R3 connection to Bluetooth HC-05

\subsubsection{Led Lighting Controller}

It can receive a wireless signal represented one or more commands transmitted over a Bluetooth wireless network from a Bluetooth - supported device to the led lighting controller. [23].

\subsubsection{Humidity and Temperature Sensor}

Humidity and Temperature Sensor monitors real time Humidity and temperature data from one room constantly to keep the room Humidity and temperature at the required level. DHT11 digital humidity and temperature sensor is a binary Sensor consist of a calibrated digital signal about humidity and temperature. The humidity and temperature sensor has high reliability. Has a humidity measurement error up to: $5 \%$, which Record humidity measurements from $20 \%$ to $95 \%$. In temperature Record measurements from zero to 50, measurement error: 2 degrees [24]. Figure 5 shows the Arduino UNO R3 connection with DHT11 and crystal led screen.

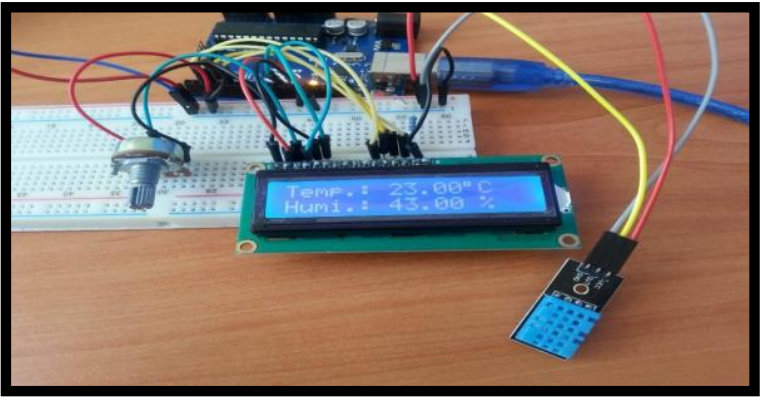

Fig 5. The Arduino UNO R3 connection with DHT11 and crystal led screen

When the room temperature becomes greater than the setting temperature, the microcontroller operates the air-condition to restore the temperature.

\subsubsection{Motion Sensor}

It is often called as PIR, "Passive Infrared", "Pyro electric", or "IR motion".

PIRs are an electronic devices used to detect moving human in or out of the sensors range. They are made of a pyro electric sensor. They are used to detect infrared radiation wavelength. Figure 6 shows the Arduino UNO R3 connection with PIR.

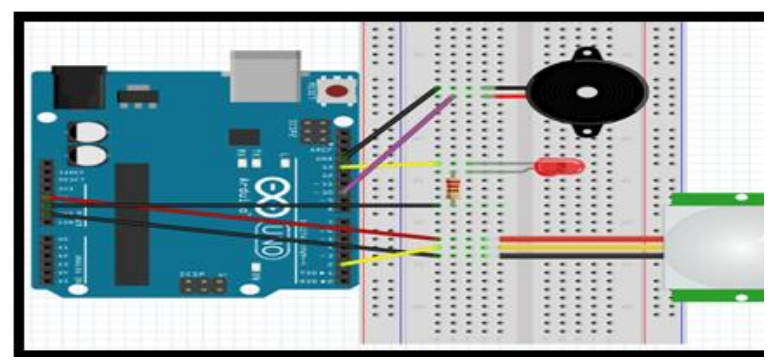

Fig 6. The Arduino UNO R3 connection with PIR

When PIR sensor detects the motion inside home, the signal is sent to microcontroller. Microcontroller make as the following: the first act is turn on the light and two act is Turn on the buzzer device.

\subsubsection{Gas / Smoke sensor}

MQ-2 Smoke Sensor has been used to detect smoke. If there is a smoke in home the Smoke Sensor will Feel it then send signal to the microcontroller to turn the fan on in order to take smoke out of home, and Turn on the buzzar device. Figure 7 shows the Arduino UNO R3 connection with MQ-2 Smoke Sensor.

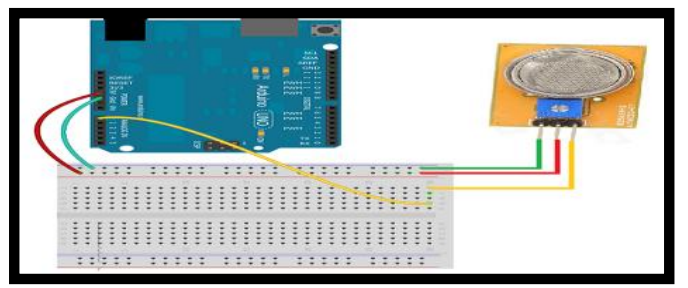

Fig 7. The Arduino UNO R3 connection with MQ-2 Smoke Sensor

\subsubsection{Servo motor}

Door and window opening and closing can be controlled by servomotor. Figure 8 shows the Arduino UNO R3 connection with servomotor. 


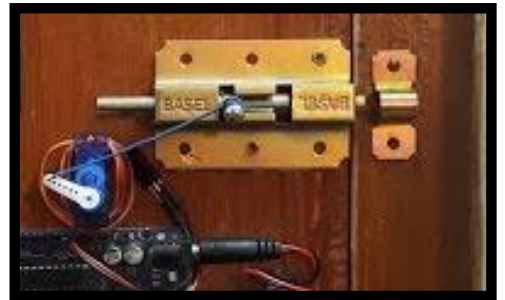

Fig 8. The Arduino UNO R3 connection with servomotor

Figure 9 shows Flow chart for suggest smart lab

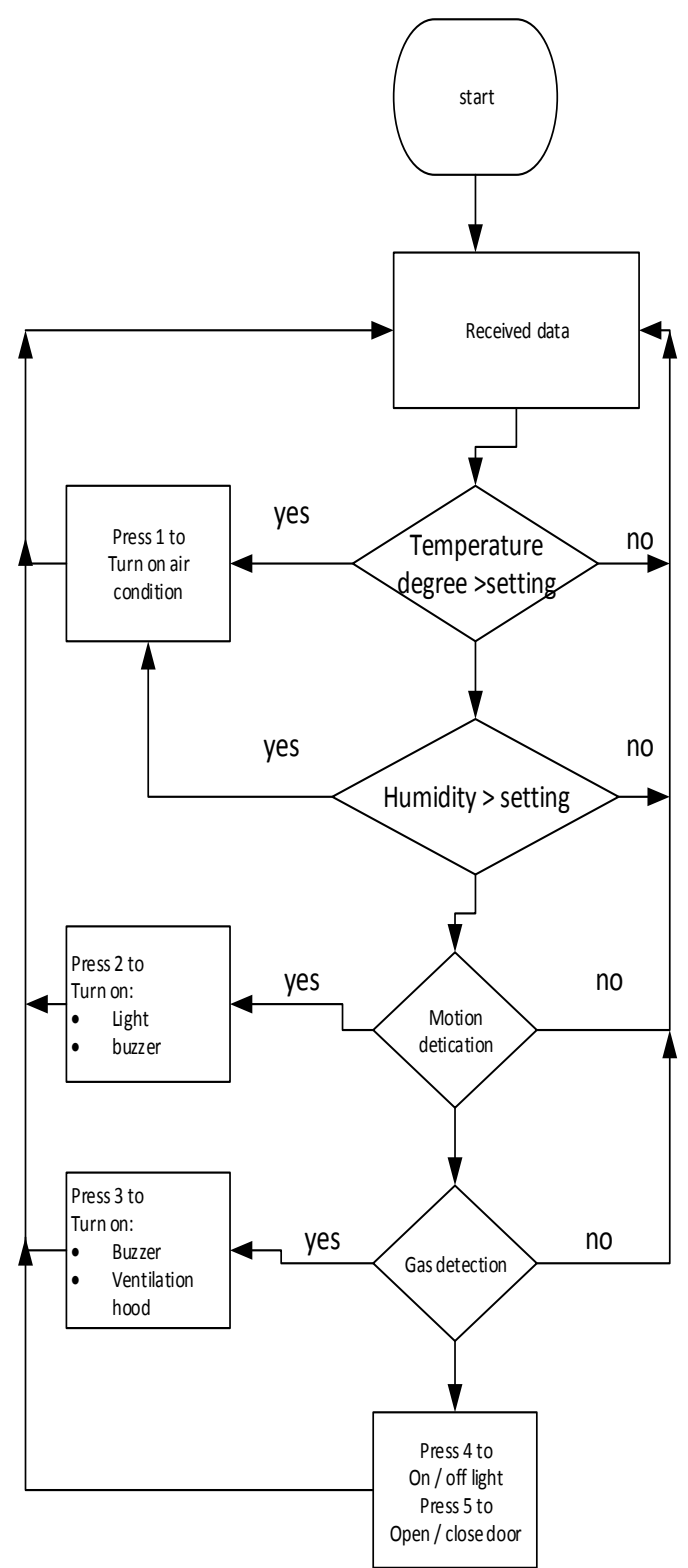

Fig.9. Flow chart for suggest smart laboratory

\subsubsection{Android Application}

Can be controlled of smart Home using Android App. it is a software for mobile devices can be content an O.S, key applications and middleware. We are using open source android platform in this automation system. Here the android application and microcontroller are connected via Bluetooth unit. If the user is authorized then he turn ON Bluetooth, then display the list of some devices for serial connection, to control in make it on or off. The Figure10 shows Screenshots of the android application.

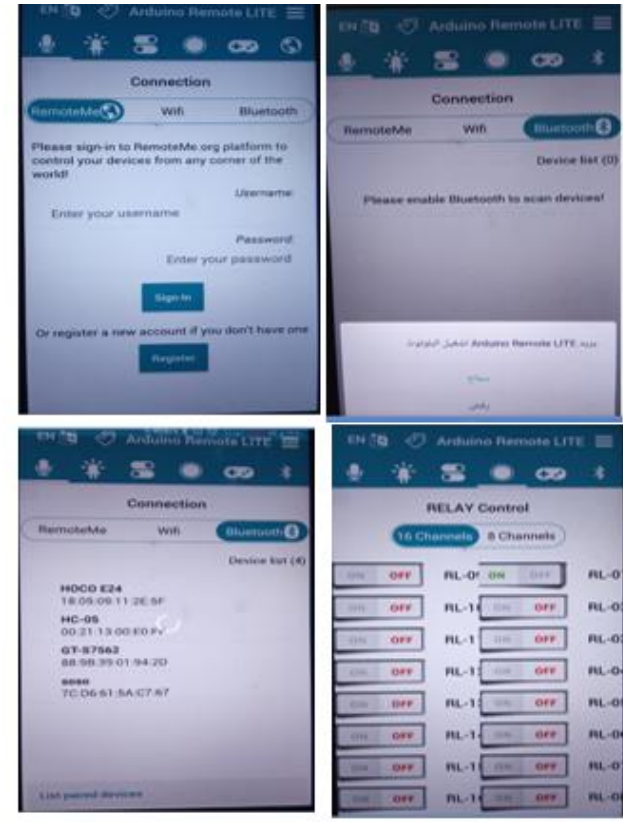

Fig 10. Screenshots of the android application

\section{The METHOD \& RESULTS}

\subsection{Sample}

To collect experimental data, a Likert questionnaire was used for 35 teachers and Laboratory Secretary in five secondary schools in Damietta. 
Table.1 The mean, standard deviation, Chi Squared and Reliability for constructs

\begin{tabular}{|c|c|c|c|c|}
\hline Constructs & The Question & mean $\pm($ SD) & $\begin{array}{l}\text { Cronbach's } \\
\text { Alpha }\end{array}$ & $\begin{array}{c}\text { Chi } \\
\text { Squared }^{\mathrm{a}}\end{array}$ \\
\hline \multirow[t]{5}{*}{$\begin{array}{l}\text { suitability of } \\
\text { using the app }\end{array}$} & Do you think the interface design is beautiful? & $2.04 \pm(, 73)$ & $.816{ }^{* *}$ & $2.48^{* *}$ \\
\hline & Is it easy for you to work with the Android app? & $2.04 \pm(, 99)$ & & $2.48^{* *}$ \\
\hline & Is it easy for you to understand the user interface? & $2.14 \pm(, 94)$ & & $9.92^{* *}$ \\
\hline & Are the colors used nice for you? & $2.14 \pm(, 94)$ & & $2.32^{* * *}$ \\
\hline & Is the use of the application flexible and comfortable? & $1.65 \pm(.63)$ & & $2.48^{* *}$ \\
\hline \multirow[t]{3}{*}{ needs of user } & $\begin{array}{l}\text { Can the application be used at any time and } \\
\text { anywhere? }\end{array}$ & $2.97 \pm(1.27)$ & $.805^{* *}$ & $3.92^{* *}$ \\
\hline & $\begin{array}{l}\text { Does the application increase the opportunities for } \\
\text { exchanging information between teachers and } \\
\text { students? }\end{array}$ & $2.97 \pm(1.27)$ & & $2.39^{* * *}$ \\
\hline & Did the application achieve the required satisfaction? & $2.18 \pm(1.00)$ & & $9.72^{* *}$ \\
\hline \multirow{8}{*}{$\begin{array}{l}\text { work of the } \\
\text { components in } \\
\text { the system }\end{array}$} & Bluetooth unit & $2.00 \pm(.73)$ & $.912^{* *}$ & $1.28^{\mathrm{a}^{* *}}$ \\
\hline & Lighting sensor & $2.00 \pm(1.00)$ & & $9.68^{* *}$ \\
\hline & Crystal led screen & $1.76 \pm(.77)$ & & $2.24^{* *}$ \\
\hline & Servo motor & $2.28 \pm(.79)$ & & $2.96^{* *}$ \\
\hline & Temperature sensor & $2.12 \pm(.88)$ & & $1.52^{* *}$ \\
\hline & Humidity sensor & $1.76 \pm(.72)$ & & $3.44^{* *}$ \\
\hline & Motion sensor & $2.04 \pm(.88)$ & & $1.040^{* *}$ \\
\hline & Gas sensor & $1.92 \pm(.75)$ & & $1.52^{* *}$ \\
\hline
\end{tabular}

\footnotetext{
a chi-squared used to test time trends; $d f=2$
}

\subsection{Procedure}

Since there are no reliable, tools for evaluating sample opinions on appropriateness the lab environment using

Internet of Things, we prepared a questionnaire after reviewing the literature. The reliability measure of the

Questionnaire was used Cranach's $\alpha$ ( $\geq 0.96)$, was .844, Therefore, the reliability of the questionnaire was acceptable for the application. The questionnaire consists of three constructs, constructs comprising Seventeen components that were prepared for them in Arabic language. The first axis is the suitability of using the app (SUA), the second is the needs of user (ND), and the third is the work of the components in the system (WCS) in the system. The participants were informed of the aim of the study and the definition of the Internet of things. Each participant was given a paper explaining the objectives of the study, the researchers and the methodology.

\subsection{Results}

Reliability of the elements of the questionnaire was calculated to ensure the existence of correlations and questions related to the tool as a whole. The internal consistency value of the components of the constructs ranged between .912 and .805 These values generally refer to an acceptable value for the reliability and Valid of the tool (at p <0.05). The results indicated that there is a strong correlation between the elements of constructs. Table 1 shows the mean, standard deviation, and Reliability for constructs. 
The mean of sample responses relative to the constructs (suitability of using the app)

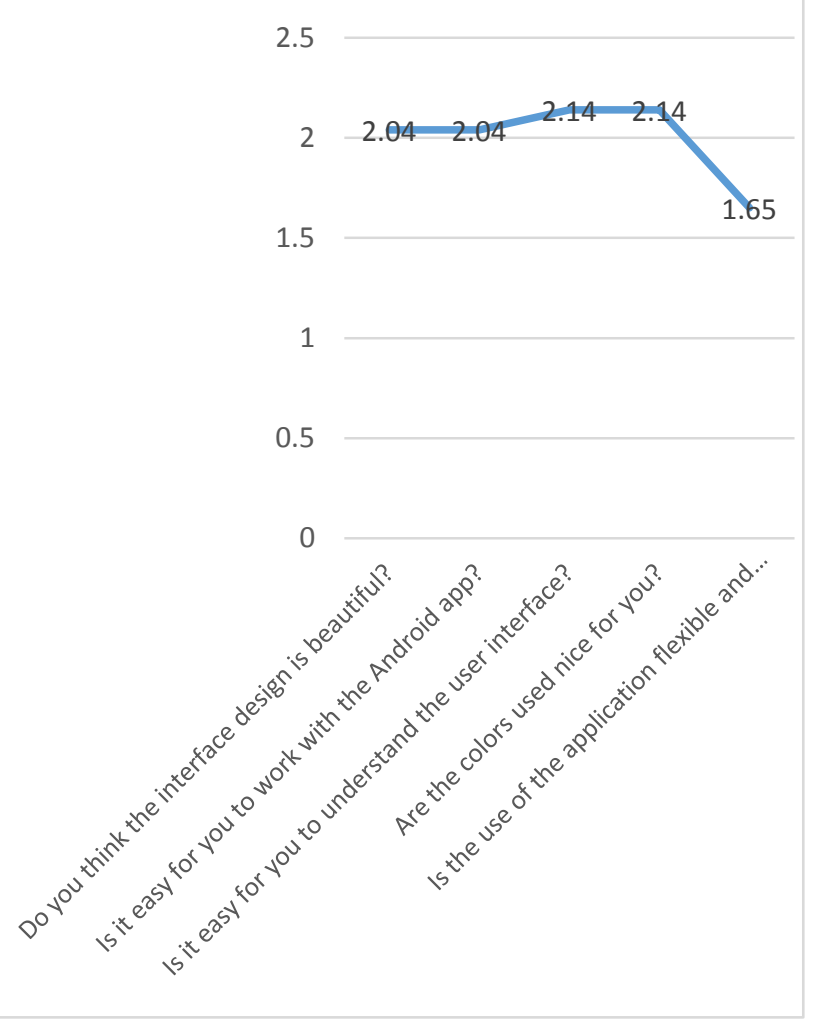

Fig.11 The mean of sample responses relative to the constructs (suitability of using the app)

As a result conduct on Table 8 and Fig. 10; five questions were answered with a mean of 2.002. One question did not reach 2.002 .

The highest value was for the third and fourth questions and this indicates that the colors used in the application interface were comfortable for them and helped participants understand the user interface easily. The minimum value was the fifth question (Is the use of the application flexible and comfortable?) with an average of 1.65 less than 2.002 and this reflects a defect in the design of the application interface.

\section{The mean of sample responses relative to the constructs (needs of user)}

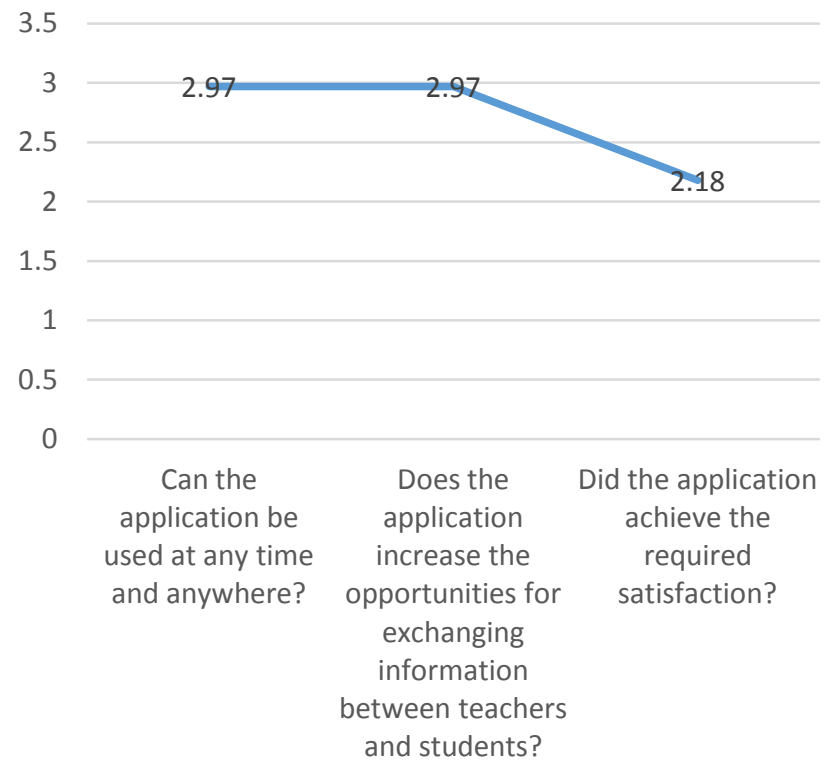

Fig.12 The mean of sample responses relative to the constructs (needs of user)

Based on the participants' feedback in Figure 11, the proposed application can predict whether the smart lab room is healthy and suitable for practical lessons. In order to help save time to increase students' better learning opportunities. Using the app increases opportunities for exchanging information between teachers and students by $2.97 \pm(1.27)$, which is the highest value in this construct.

The mean of sample responses relative to the constructs ( work of the components in the system)

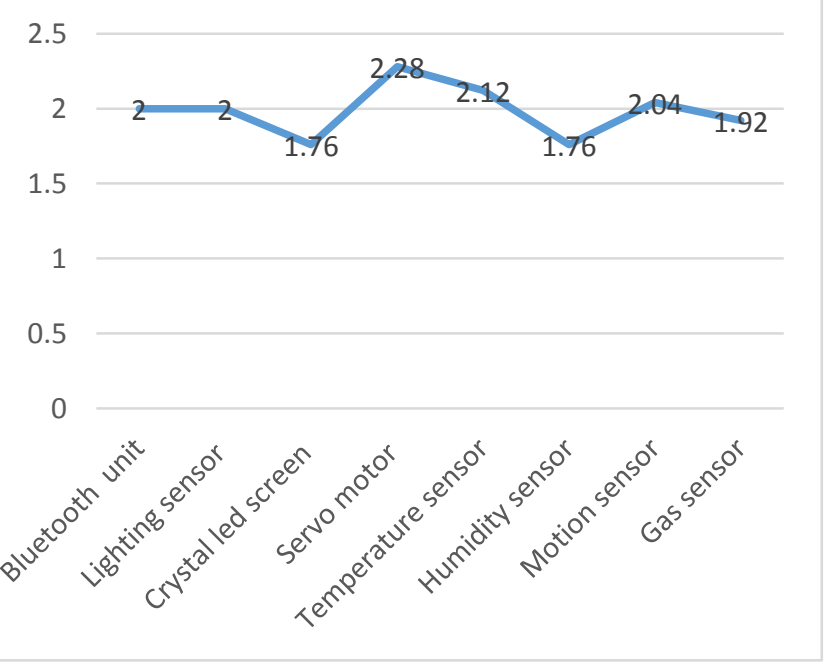

Fig.13 The mean of sample responses relative to the constructs (work of the components in the system) 
Based on the results in the table and figure, the participants have predicted what the iot can add to control in the lab room. The results revealed that the proposed system could achieve the following:

- can be Control the opening and closing of the leds using android application where the user can open and close the leds even from outside the laboratory .Lighting sensor ( $2.00 \pm(1.00))$

- Display temperature and humidity on the illuminated screen and when the reading becomes higher than the normal rate is turned on the air conditioner. Temperature sensor $(2.12 \pm(.88))$, Humidity sensor $(1.76 \pm(.72))$, and Crystal led screen $(1.76 \pm(.77))$.

- Movement in the surrounding area was explored by infrared and in the case of a feeling of movement in the laboratory during the other than the official working hour's triggers an alarm indicating the presence of danger. Motion sensor (2.04 $\pm(.88))$.

- The gas sensor, an alarm is triggered when there are fires or flammable gas, and a fan is turn on to expel gas outside the laboratory .Gas sensor (1.92 (.75)).

- Windows and doors, to protect the laboratory against theft. Servomotor $(2.28 \pm(.79))$.

\section{CONCLUSIONS AND FUTURE WORK}

In this paper, a smart laboratory system is proposed and successfully implemented. The system can control lighting and closing leds, temperature and humidity monitoring, fire control, gas density, burglar alarm, door and window control at laboratory and has an infrared sensor to ensure family security. Features such as laboratory control, low cost, and monitoring system using Android based Smart phone make the proposed system Useful and useful. Future works will focus on implementation of voice commands for controlling the application via voice.

\section{REFERENCES}

[1] Gubbi, J., Buyya, R., Marusic, S., \& Palaniswami, M. (2013). Internet of Things (IoT): A vision, architectural elements, and future directions. Future generation computer systems, 29(7), 1645-1660.

[2] Mahmoud, M. S., \& Mohamad, A. A. (2016). A study of efficient power consumption wireless communication techniques/modules for internet of things (IoT) applications.

[3] Qun, Z., \& Weihua, X. (2013, September). The construction of knowledge sharing platform in universities under the ubiquitous network environment. In 2013 Fourth International Conference on Emerging Intelligent Data and Web Technologies (pp. 104-108). IEEE.

[4] Majstorovic, V., \& Rakic, K. (2017, January). Internet of Things and Social Media: Tools of a Successful Information Organization. In Proceedings of the 28th DAAAM International Symposium (pp. 0295-0298).

[5] Al Marouf, A., Islam, S., \& Chakraborty, N. R. IoT-based Smart Meeting Room Weather Detection System using Arduino and Relative Sensors. International Journal of Computer Applications, 975, 8887.

[6] Albino, V., Berardi, U., \& Dangelico, R. M. (2015). Smart cities: Definitions, dimensions, performance, and initiatives. Journal of urban technology, 22(1), 3-21

[7] Rosas, S. V., Flores, D. F. O., Vallejo, A., \& Hernández, U. A. H. S. (2019). Smart cities of the future: An interdisciplinary literature review. Dimensions and proposed characteristics. International Journal of Advanced Engineering, Management and Science (IJAEMS), 587.

[8] Kaur, M. J., \& Maheshwari, P. (2016, March). Building smart cities applications using IoT and cloud-based architectures. In 2016 International Conference on Industrial Informatics and Computer Systems (CIICS) (pp. 1-5). IEEE.

[9] ElKamchouchi, H., \& ElShafee, A. (2012, November). Design and prototype implementation of SMS based home automation system. In 2012 IEEE International Conference on Electronics Design, Systems and Applications (ICEDSA) (pp. 162-167). IEEE.

[10] Vishnu, A., Samuel, A. P., Jishnu, V. R., Kiran, J., \& Ravikumar, M. (2016). Home Automation Prototype using IoT. ICCIT16@ CiTech, Bengaluru.

[11] Mir-Nasiri, N., Siswoyo, H., \& Ali, M. H. (2018). Portable autonomous window cleaning robot. Procedia computer science, 133, 197-204.

[12] Psillos, D., \& Niedderer, H. (2002). Issues and questions regarding the effectiveness of labwork. In Teaching and learning in the science laboratory (pp. 21-30). Springer, Dordrecht.

[13] Berry, A., Gunstone, R., Lougharan, J., \& Mulhall, P. (2002). Using laboratory work to teach about the practice of science. Research in Science Education-Past, Present and Future, Part 6, 313-318.

[14] O’Sullivan, C. (2007). Teaching Heat Transfer to Engineering Students-a course of computer-based handson activities. In International Conference on Engineering Education (pp. 1-5)

[15] Poongothai, M., Subramanian, P. M., \& Rajeswari, A. (2018, April). Design and implementation of IoT based smart laboratory. In 2018 5th International Conference on Industrial Engineering and Applications (ICIEA) (pp. 169-173). IEEE.

[16] Gavrilov, A. V., Novitskaya, Y. V., \& Yatsevich, T. A. (2014). Towards a smart school laboratory. DIVAI, 10, 65-74.

[17] Yin, H. (2015). Smart Lab Technologies. In Handbook of Mobile Teaching and Learning (pp. 1-11). SpringerVerlag Berlin Heidelberg.

[18] Cherian, M., \& Hitesh Kumar, P. (2014). Implementation of a Secure and Smart Lab with Wireless Sensor Network. International Journal of Science and Research, 3(6)

[19] Al-Fuqaha, A., Guizani, M., Mohammadi, M., Aledhari, M., \& Ayyash, M. (2015). Internet of things: A survey on enabling technologies, protocols, and applications. IEEE communications surveys \& tutorials, 17(4), 2347-2376.

[20] Yassein, M. B., \& Shatnawi, M. Q. (2016, September). Application layer protocols for the Internet of Things: A survey. In 2016 International Conference on Engineering \& MIS (ICEMIS) (pp. 1-4). IEEE. 
International Journal of Computer Applications (0975 - 8887)

Volume 175-No.19, September 2020

[21] https://www.arduino.cc/Main/arduinoBoardUno.html.

[22] Kumar, C. B., Kline, P. J., \& Thompson, T. J. (2004). Bluetooth application programming with the Java APIs. Morgan Kaufmann.
[23] Lighting system controller configured to be remotely controlled via a Bluetooth-enabled wireless device for controlling outdoor LED lighting. U.S. Patent No 10,165,659, 2018.

[24] DHT11 Product Manual, www.geekbotelectronics.com. 